Diabetologe 2020 $16: 627-629$

https://doi.org/10.1007/s11428-020-00676-x

(c) Springer Medizin Verlag $\mathrm{GmbH}$, ein Teil von Springer Nature 2020

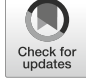

\author{
A. Glaser ${ }^{1} \cdot$ M. Hrabě de Angelis ${ }^{1,2,3}$ \\ 'Deutsches Zentrum für Diabetesforschung (DZD), Neuherberg, Deutschland \\ ${ }^{2}$ Institut für Experimentelle Genetik, Helmholtz Zentrum München - Deutsches Forschungszentrum für \\ Gesundheit und Umwelt, Neuherberg, Deutschland \\ ${ }^{3}$ Lehrstuhl für Experimentelle Genetik, Technische Universität München, München, Deutschland
}

\title{
Neues aus der Diabetesforschung
}

Neue Wege für eine präzise Diabetesprävention und -therapie aufzuzeigen, ist das wichtigste Ziel des Deutschen Zentrums für Diabetesforschung (DZD), in dem deutschlandweit exzellente Expertinnen und Experten aus Grundlagenforschung, Epidemiologie, Versorgungsforschung und Klinik zusammenarbeiten. Aktuelle Forschungsergebnisse aus dem DZD und anderen internationalen Diabetesforschungsgruppen zeigen, dass wir diesem Ziel einen großen Schritt näherkommen konnten. Die interdisziplinäre, vertrauensvolle Zusammenarbeit in translationalen Forschungsteams, die Durchführung großer Multizenterstudien sowie Kooperationen mit internationalen Arbeitsgruppen führten $\mathrm{zu}$ wegweisenden, neuen Entwicklungen, von denen wir einige in diesem Heft vorstellen möchten. Uns ist es zudem wichtig, dass die Bevölkerung regelmäßig und verständlich über relevante Erkenntnisse informiert wird, um so die Gesundheitskompetenz zu erhöhen. Lesen Sie dazu mehr in dem Beitrag zu dem neuen nationalen Diabetesinformationsportal www.diabinfo.de (Brandt et al.).

\section{Einteilung für 5 Diabetes- Subtypen ermöglicht präzisere Behandlung}

In der derzeitigen Klassifikation der DDG (Deutsche Diabetes Gesellschaft) sind jenseits von Gestationsdiabetes und Sonderformen mit bekannten Ursachen 2 Diabetestypen beschrieben, der Typ1- und der Typ-2-Diabetes, welche sich insbesondere hinsichtlich ihrer Pathophysiologie unterscheiden. Resultate von Studien aus Skandinavien deuten darauf hin, dass es mehrere Untertypen (Cluster) des Diabetes gibt. Nun wurde ausgehend von großen internationalen Kohorten eine neue Einteilung der Diabetestypen vorgeschlagen. Wie Zaharia et al. in diesem Heft beschreiben, ermöglicht die neue Klassifikation in 5 anstelle von wie bisher 2 Diabetestypen (Cluster) nicht nur eine differenzierte Einteilung und eine damit verbundene präzisere Therapie der Patientinnen und Patienten mit Diabetes, wie kürzlich von einer schwedischen Forschungsgruppe berichtet, sondern auch eine gezieltere Prävention oder frühzeitige Behandlung von diabetesbedingten Folge- und Begleiterkrankungen [1].

\section{》) Die neue Einteilung ermöglicht eine individuellere Therapie der Patienten mit Diabetes}

Ergebnisse aus der deutschen Diabetesstudie deuten darauf hin, dass, je nachdem in welches Cluster eine Patientin bzw. ein Patient eingeordnet werden kann, auch die Risiken für Komorbiditäten und Komplikationen unterschiedlich hoch sind [2]. Die Entdeckung von Clustern im Prädiabetesbereich und die sich daraus ergebenden neuen Möglichkeiten einer präziseren Prävention wurden von Andreas Fritsche und DZD-Kollegen bereits im Mai in dem Themenheft Prävention dieses Journals aufgezeigt hierzu wird demnächst eine Originalarbeit in dem renommierten Journal Nature Medicine erscheinen.

\section{Intervallfasten - Effekte auf Stoffwechsel und Insulinsensitivität}

Übergewicht und Adipositas erhöhen die Risiken für Stoffwechselstörungen und können zu einem Typ-2-Diabetes führen. In diesen Fällen ist die Gewichtsabnahme ein wesentlicher Faktor für die Prävention dieser Stoffwechselerkrankung. Als vielversprechende Maßnahme wird Intervallfasten in den Medien hochgelobt. Welche Mechanismen beim intermittierenden Fasten derzeit bekannt sind, stellen Schürmann et al. in diesem Heft ausführlich vor. So lieferten Tierund Humanstudien Hinweise darauf, dass Intervallfasten bei Adipositas zu einer Verringerung der Körperfettmasse sowie zu Verbesserungen der Stoffwechselparameter und der Insulinsensitivität führt. Untersuchungen im Tiermodell ergaben, dass diese Art des Fastens auch den Fettgehalt der Bauchspeicheldrüse schrumpfen lässt [3].

\section{Materner Metabolismus und Diabetesentstehung}

Ein Forschungsschwerpunkt im DZD ist die Untersuchung der Frage, welchen Einfluss die Insulinwirkung bzw. die Insulinresistenz im Gehirn auf die Diabetesentwicklung nehmen. Reagiert das Gehirn nur wenig oder gar nicht auf Insulin, kann dies zur Entstehung von Fettleibigkeit beitragen [4]. Wann sich im Lauf des Lebens die Insulinempfindlichkeit im Gehirn von Übergewichtigen verschlechtert, ist noch unbekannt. Möglicherweise geschieht dies schon sehr früh. Die Hirnfunktion des ungeborenen Kindes wird anscheinend vom 
Stoffwechsel der Mutter beeinflusst. Das könnte Folgen für das spätere Diabetes- und Übergewichtsrisiko des Kindes haben [5]. Einen guten Überblick über Studien, in welchen die Einflüsse des maternen Metabolismus auf die fetale Entwicklung des zentralen und des autonomen Nervensystems untersucht wurden, geben Schlegel et al.

\section{Aktuelle Situation in der Früherkennung und Prävention des Typ-1-Diabetes}

Auch bezüglich der Erforschung der Entstehung des Typ-1-Diabetes sowie der Entwicklung neuer Präventionsansätze wurden in den vergangenen Jahren dank auch Kolleginnen und Kollegen des DZD Fortschritte erzielt. Anhand großer prospektiver Geburtskohorten wurden entscheidende Fortschritte im Verständnis für die Entstehung und den natürlichen Verlauf dieser chronischen Autoimmunerkrankung erzielt.

Der Typ-1-Diabetes kann heute frühzeitig durch den Nachweis spezifischer Inselantikörper diagnostiziert werden, lange bevor es $\mathrm{zu}$ Veränderungen des Glukosestoffwechsels oder gar zu Symptomen kommt. Das bessere Verständnis der komplexen Vorgänge, die zur Fehlleitung des Immunsystems und zur fortschreitenden Zerstörung von Betazellen führen, eröffnet auch neue Ansatzpunkte, um das Entstehen von Autoimmunität zu vermeiden. Aktuell werden in mehreren deutschen Bundesländern Screeningprogramme durchgeführt, um Kinder mit einem erhöhten genetischen Risiko früh zu erkennen.

\section{) Mittels Screening können Kinder mit einem erhöhten Typ- 1-Diabetes-Risiko identifiziert werden}

In der randomisierten, kontrollierten Doppelblindstudie POInT („primary oral insulin trial") erhalten Kinder mit einem erhöhten Risiko für Typ1-Diabetes täglich eine kleine Menge Insulinpulver bzw., zur Kontrolle, ein Scheinmedikament mit einer Mahlzeit.
Ziel ist es, das Immunsystem zu trainieren, körpereigenes Insulin zu tolerieren [6]. In dieser und weiteren Studien wird derzeit untersucht, ob sich durch eine Art Desensibilisierung bei Risikopatientinnen und -patienten der Ausbruch der Autoimmunerkrankung verzögern oder gar vermeiden lässt. Ein übersichtliches Update zu den spannenden neuen Aktivitäten und Erkenntnissen zur Früherkennung und Prävention des Typ-1Diabetes finden Sie in dem Artikel von Marcus et al.

\section{(Xeno)-Transplantationen und künstliches Pankreas}

Beim Typ-1-Diabetes sowie im fortgeschrittenen Stadium des Typ-2-Diabetes gehen die insulinproduzierenden Betazellen in der Bauchspeicheldrüse zugrunde. Die derzeit einzig klinisch verfügbaren Methoden zur Betazellersatztherapie sind Pankreas- und Inselzelltransplantationen. Eine breitere Anwendung ist aber durch die geringe Verfügbarkeit von Spenderorganen begrenzt. Zudem sind Transplantationen aufgrund der Notwendigkeit einer dauerhaften Immunsuppression zur Abstoßungsprophylaxe für viele Patientinnen und Patienten nicht geeignet.

In ihren Artikel zeigen Ludwig $u$. Wolf Zukunftsperspektiven der Transplantation auf. In der „Betacell-Academy“ arbeiten Forschende des DZD u. a. daran, Stammzellen oder tierische Gewebe (Xenotransplantation) als Quelle für Transplantate $z u$ nutzen. Ihnen gelang es, Schweine genetisch so $\mathrm{zu}$ verändern, dass die Inselzellen das Immunsystem lokal hemmen [7]. Auch innovative Konzepte der Mikro- oder Makroverkapselung der Inselzellen können helfen, Abstoßungsreaktionen zu vermeiden [8]. Diese vielversprechenden Konzepte können künftig neue Wege in der Transplantation eröffnen.

\section{Ausblick}

In den vergangenen Jahren konnten in der Diabetesforschung entscheidende Fortschritte zum Wohle der Patientinnen und Patienten erreicht werden, aber es gibt noch viel zu tun. Hierbei wird die Digitalisierung mit einer dringend notwendigen umfassenden und nachhaltigen Erschließung, Verknüpfung und Nutzung von Datenbeständen aus Grundlagenforschung, klinischer Forschung und der Versorgung von Patientinnen und Patienten eine entscheidende Rolle spielen. Innovative Methoden aus dem Bereich ,artificial intelligence “ und „machine learning“ können dann helfen, präzise Präventions- und Therapiekonzepte zu entwickeln.

Abschließend möchten wir betonen, dass es für die zukünftige translationale Diabetesforschung wichtig ist, junge Wissenschaftlerinnen und Wissenschaftler sowie Ärztinnen und Ärzte für die Diabetesforschung zu begeistern. Dazu ist es notwendig, ihnen über den Karriereweg des „Medical Scientist“ und des „Clinician Scientist“ sowie den Erhalt der klinischen Lehrstühle in der Diabetologie Perspektiven für die Forschung und ihren Berufsweg zu bieten.

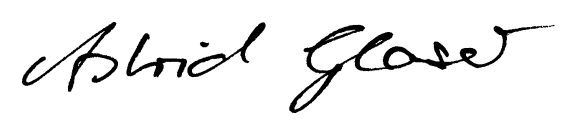

Dr. Astrid Glaser

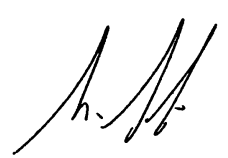

Prof. Dr. Dr. h.c. mult. Martin Hrabě de Angelis

\section{Korrespondenzadresse}

Prof. Dr. Dr. h.c. mult. M. Hrabě de Angelis

Deutsches Zentrum für Diabetesforschung (DZD)

Ingolstädter Landstr. 1, 85764 Neuherberg,

Deutschland

niesing@dzd-ev.de

Interessenkonflikt. A.Glaser und M. Hrabě de Angelis geben an, dass kein Interessenkonflikt besteht.

\section{Literatur}

1. AhlkvistEetal (2018) Novel subgroups of adult-onset diabetes and their association with outcomes: a data-driven cluster analysis of six variables. Lancet Diabetes Endocrinol 6(5):P361-369. https:// doi.org/10.1016/S2213-8587(18)30051-2

2. Zaharia OP et al (2019) Risk of diabetes-associated diseases in subgroups of patients with recentonset diabetes: a 5-year follow-up study. Lancet 
Diabetes Endocrinol 7(9):684-694. https://doi. org/10.1016/s2213-8587(19)30187-1

3. Quiclet $C$ et al (2019) Pancreatic adipocytes mediate hypersecretion of insulin in diabetessusceptible mice. Metabolism 97:9-17. https:// doi.org/10.1016/j.metabol.2019.05.005

4. Kullmann S et al (2020) Central nervous pathways of insulin action in the control of metabolism and food intake. Lancet Diabetes Endocrinol 8(6):524-534. https://doi.org/10.1016/S22138587(20)30113-3

5. Linder K et al (2015) Gestational diabetes impairs human fetal postprandial brain activity. I Clin Endocrinol Metab 100(11):4029-4036. https://doi. org/10.1210/jc.2015-2692

6. Winkler $C$ et al (2019) Identification of infants with increased type 1 diabetes genetic risk for enrollment into Primary Prevention Trials-GPPAD02 study design and first results. Pediatr Diabetes 20(6):720-727. https://doi.org/10.1111/pedi. 12870

7. Kemter $E$ et al (2018) Will genetic engineering carry xenotransplantation of pig islets to the clinic? CurrDiabRep 18(11):103. https://doi.org/10.1007/ s11892-018-1074-5

8. Ludwig B et al (2017) Favorable outcome of experimental islet xenotransplantation without immunosuppression in a nonhuman primate model of diabetes. Proc Natl Acad Sci U S A 114(44):11745-11750. https://doi.org/10.1073/ pnas. 1708420114

\section{Jahre DPV-Register}

Wo stehen wir heute in der Versorgung von Kindern und Jugendlichen mit Diabetes?

Seit nunmehr 25 Jahren besteht mit dem DPV-Register für Kinder, Jugendliche und junge Erwachsene eine bundesweite Initiative zur Qualitätssicherung, die individuelle Patientendaten wie Geschlecht, Manifestationsalter und Diabetestyp multizentrisch erfasst. „Durch diese Datensammlung von inzwischen über 92.000 Kindern und Jugendlichen konnten wir über die Jahre insgesamt eine Verbesserung in der Stoffwechseleinstellung der Patientinnen und Patienten feststellen", bilanziert DDG Experte Professor Dr. med. Reinhard Holl, Vorsitzender der AG Diabetologie Baden-Württemberg e.V.. „Darüber hinaus liefert es uns einmalige Erkenntnisse zu seltenen Diabetesformen wie dem Neugeborenen-Diabetes sowie Diabetes als Medikamenten-Nebenwirkung oder aktuell zu COVID-19-Auswirkungen auf Diabetespatienten."

Insgesamt liegt in Deutschland eine gute medizinische Versorgungslage vor und die Therapieergebnisse haben sich in den vergangenen Jahrzehnten deutlich verbessert. Eine wichtige Entwicklung der vergangenen Jahre war der Einzug neuer Technologien wie Insulinpumpen und die kontinuierliche Glukosemessung in die Praxis. Bei immer jüngeren Patienten wurden diese erfolgreich eingesetzt: Während für das Behandlungsjahr 2015 noch bei 932 Kindern und Jugendlichen mit Diabetes Typ 1 eine sensorunterstützte Pumpentherapie (SUP) entweder mittels kontinuierlicher Glukosemessung (CGM) oder Flash Glucose Monitoring (FGM) dokumentiert war, waren es drei Jahre später bereits 10.180 Patientinnen und Patienten.

„Aber nicht alles wird besser: Die Zahl gefährlicher Stoffwechselentgleisungen mit diabetischer Ketoazidose hat leider nicht abgenommen", erklärt Holl, Leiter der Arbeitsgruppe Computergestütztes Qualitätsmanagement in der Medizin im epidemiologischen Institut der Universität UIm. Noch immer besteht eine diabetische Ketoazidose bei jedem fünften Patienten unter 20 Jahren bei Diagnosestellung des Typ-1-Diabetes. „Höchste Priorität hat daher die Früherkennung: Vermehrtes Trinken, häufiges Wasserlassen und Gewichtsabnahme sind die ersten Anzeichen einer Diabeteserkrankung", erklärt Professor Dr. med. Andreas Neu, Vizepräsident der DDG. Sind diese Symptome vorhanden, sei ein umgehender Arztbesuch zwingend notwendig um schwere Stoffwechselentgleisungen, die lebensbedrohlich werden können, zu verhindern, so der Kommissarische Ärztliche Direktor an der Klinik für Kinder- und Jugendmedizin am Universitätsklinikum Tübingen.

Hinzu kommt die steigende Zahl von Typ-2Diabetes-Patienten in dieser Altersgruppe. „Viele Kinder und Jugendliche in Deutschland haben heute Übergewicht oder sind adipös. Der Typ-2-Diabetes, der früher als „Altersdiabetes" bezeichnet wurde, wird heute auch bei manchen schwer adipösen Minderjährigen diagnostiziert", führt Holl aus. In Deutschland sind derzeit laut DPV-Register und anderen Datenquellen rund 1.000 Jugendliche betroffen, darunter knapp 70 Prozent Mädchen. 30 Prozent der Betroffenen haben einen Migrationshintergrund.

Auch regionale Unterschiede sind durch die DPV-Datenerfassung abbildbar. Unter den 16 Bundesländern schwankt beispielsweise die Stoffwechseleinstellung bei Kindern und Jugendlichen mit Typ-1-Diabetes zwischen 7,5 Prozent und 8,4 Prozent. Schwere Unterzuckerungen (Hypoglykämien) treten je nach Bundesland bis zu 3,5-mal häufiger auf.

Am DPV-Register beteiligen sich derzeit 283 pädiatrische und 205 internistische Einrichtungen. 440 Zentren sind aus Deutschland, 43 aus Österreich, vier aus der Schweiz und eines aus Luxemburg. Inzwischen wurden etwa 300 Publikationen zur DPV-Initiative veröffentlicht. Insgesamt sind mittlerweile knapp 650.000 Menschen mit Diabetes im DPV-Register erfasst.

Quelle: www.deutsche-diabetesgesellschaft.de 